\title{
Enfoques, evolución y afrontamiento del envejecimiento demográfico en Cuba
}

\author{
Héctor Demetrio Bayarre Vea, ${ }^{1}$ María Esther Álvarez Lauzarique, ${ }^{1}$ Julia Silvia \\ Pérez Piñero, ${ }^{1}$ Kenia Almenares Rodríguez, ${ }^{1}$ Aida Rodríguez Cabrera, ${ }^{1}$ \\ María del Carmen Pría Barros, ${ }^{1}$ Lilliams Rodríguez Rivera, ${ }^{2}$ Alberto Fernández \\ Seco $^{3}$ y Ariadna Corral Martín ${ }^{1}$
}

Forma de citar

Bayarre Vea HD, Álvarez Lauzarique ME, Pérez Piñero JS, Almenares Rodríguez K, Rodríguez Cabrera A, Pría Barros MC, et al. Enfoques, evolución y afrontamiento del envejecimiento demográfico en Cuba. Rev Panam Salud Publica. 2018;42:e21. https:// doi.org/10.26633/RPSP.2018.21

RESUMEN El envejecimiento demográfico en Cuba ha provocado cambios considerables en la mortalidad y la morbilidad; su tendencia creciente y alta velocidad comprometen la atención de la persona mayor, de ahí que sea un desafío para la salud pública. Este artículo, que abarca el período 19502015, tiene como finalidad exponer la situación de este fenómeno poblacional desde una visión positiva, según tres aspectos: el envejecimiento como logro, desafío y oportunidad de desarrollo; las transiciones demográfica y epidemiológica; y las políticas y estrategias para afrontarlo. La tasa general de fecundidad ha descendido desde 4,01 hasta 1,45, y la esperanza de vida al nacer y la esperanza de vida a la tercera edad se han incrementado desde 59,40 hasta 78,45 años y desde 16,04 hasta 22,58, respectivamente. La proporción de personas de 60 años o más en Cuba asciende a 19,8\%. El cuadro de morbilidad y mortalidad ha cambiado de las enfermedades infectocontagiosas a las no transmisibles. El envejecimiento en Cuba se ha producido por la reducción sostenida de la fecundidad, la mortalidad y un saldo migratorio negativo. Se discute el reto que significa la dependencia de la persona mayor y la necesidad de cuidados que requiere. Además del logro y la oportunidad de desarrollo que representa este indicador demográfico, el envejecimiento poblacional constituye un desafío para el desarrollo y la salud pública, para el que se han establecido politicas y estrategias integrales e intersectoriales encaminadas a mitigar sus efectos negativos.

Palabras clave Envejecimiento demográfico; salud pública; transición demográfica; transición de la salud; políticas públicas; Cuba.

El envejecimiento de la población cubana es un tema recurrente debido a los cambios ocurridos en las últimas décadas. Definido como el aumento de la proporción de personas mayores - por lo general de 60 años o más-, el

\footnotetext{
Escuela Nacional de Salud Pública, La Habana, Cuba. Enviar la correspondencia a Héctor Demetrio Bayarre Vea, hbayarre@infomed.sld.cu

2 Centro de Investigaciones sobre Longevidad, Envejecimiento y Salud, La Habana, Cuba. 3 Ministerio de Salud Pública, La Habana, Cuba.
}

envejecimiento es el resultado del efecto combinado de la fecundidad, la mortalidad y las migraciones, que produce cambios importantes sobre la estructura poblacional (1-3). Para explicar el proceso de envejecimiento, iniciado con la Revolución Industrial, se formularon dos teorías: la transición demográfica y la transición epidemiológica $(4,5)$.

Cuba tiene el índice de envejecimiento más alto de América Latina: 19,8\% (6). En todas las provincias, los niveles de fecundidad y mortalidad son tan bajos como en los países altamente desarrollados, ${ }^{4}$

\footnotetext{
Para los efectos de este artículo, países desarrollados son los que han logrado un alto grado de industrialización, según los principales indicadores: producto interno bruto (PIB) nominal per cápita superior a US\$25 000 en el 2005, PIB — según la paridad del poder adquisitivo per cápita - superior a US\$25 000 en el 2005 e índice de desarrollo humano superior a 0,900 en el 2004 (http://www.un.org/ en/development/desa/publications/world-population-prospects-the-2012-revision.html).
} 
de ahí que el país se ubique en la llamada etapa postransicional. El flujo migratorio negativo y el escaso desarrollo económico le confieren singularidad al caso cubano y demuestra que se puede completar la transición demográfica en tales condiciones (2).

Este artículo tiene como objetivo exponer la situación del envejecimiento en Cuba desde diferentes perspectivas de análisis, esbozar sus fundamentos teóricos y presentar un bosquejo de la política planteada para su afrontamiento efectivo, en el que el aporte del sector de la salud ha sido y es primordial.

\section{EL ENVEJECIMIENTO DEMOGRÁFICO EN CUBA: PERSPECTIVAS PARA SU ANÁLISIS}

El análisis del envejecimiento demográfico discurre, en general, a partir de sus efectos negativos: el aumento en la prevalencia de enfermedades crónicas, la dependencia de la persona mayor y su influencia sobre la familia y la comunidad, el incremento de la demanda de atención a la salud, entre otros (1). Estos elementos hacen del proceso un generador de problemas sociales y sanitarios que lo convierten en un desafío acentuado para los países en desarrollo, porque a la falta de recursos y preparación para encararlo se le adiciona su mayor celeridad, marcada por un saldo migratorio negativo $(5,7)$.

Se puede pensar que en un país con recursos limitados el incremento de la demanda de servicios de salud de alto coste constituye un reto insuperable para la sociedad. A ello podría añadirse el impacto económico negativo que ocasiona la senescencia sobre la persona mayor enferma o discapacitada y su familia, al requerir durante años cuidados especiales, medicamentos y otros útiles y servicios que les permitan mitigar los efectos de la dependencia asociados con la vejez $(1,2)$.

Sin embargo, estos elementos —que acentúan los aspectos negativos- no son los únicos que se deben considerar, en tanto no permiten ver el significado positivo del cambio en la estructura de edades de la población. Como ya se ha afirmado, al analizar el envejecimiento demográfico también se deben tomar en cuenta sus aspectos positivos, ya que prolongar la vida de las personas es un logro de la humanidad y es también una oportunidad para el desarrollo socioeconómico $(1,2)$.

La declaración por parte de las Naciones Unidas de que el envejecimiento no es un problema sino un logro, y que no es un mero asunto de seguridad y bienestar social, sino de desarrollo y política económica en su conjunto $(1,7)$, abrió un enfoque más positivo, acogido por los expertos y las autoridades cubanas. El envejecimiento de la población, por tanto, debe verse como un logro social, pues vivir más ha sido una meta de nuestra especie y en su consecución ha influido mucho el avance científico-técnico alcanzado. Es también una oportunidad para el desarrollo socioeconómico, si la sociedad aprovecha la experiencia acumulada en la preparación de las nuevas generaciones de trabajadores (transferencia) mediante el mantenimiento de un vínculo laboral en las condiciones adecuadas. Asimismo, este segmento de la población crea nuevas demandas de bienes y servicios, cuya satisfacción pudiera constituir una fuente de empleo e ingresos (1).

En los países envejecidos como Cuba es menester considerar y aprovechar las capacidades de las personas mayores como fuente de desarrollo sostenible. Las investigaciones sociales pueden ayudar a ordenar esta situación, de modo que se adecuen las condiciones laborales para este grupo de población.

\section{LAS TRANSICIONES DEMOGRÁFICA Y EPIDEMIOLÓGICA: EVOLUCIÓN Y PERSPECTIVAS}

La transición demográfica, que surge con la modernización ocurrida en los países industrializados desde finales del siglo XVIII, es el principal referente teórico para explicar el proceso de envejecimiento por el que transita el mundo. Esta teoría expone el cambio de los niveles de fecundidad y mortalidad altos o muy altos a bajos o muy bajos, situación que en un primer momento se dio en los países industrializados y que hoy se ha expandido en mayor o menor medida a todos los países (4, 8-11).

\section{La transición demográfica en América Latina y Cuba}

En América Latina y el Caribe, el envejecimiento poblacional iniciado en la década de 1970 se distingue por su mayor celeridad respecto a los países de Europa
(5), especialmente en Cuba, Argentina, Uruguay, Antillas Holandesas, Barbados, Guadalupe, Martinica y Puerto Rico, en ese orden; otros, como Haití, se encuentran en los primeros estadios de este proceso $(2,5)$.

Para ilustrar la evolución de la transición demográfica en Cuba y compararla con la de algunos países desarrollados y de América Latina resultan útiles algunos indicadores de mortalidad y fecundidad, como se ve a continuación.

La tasa general de fecundidad. Este indicador mostró una tendencia descendente entre 1950 y el 2015, con diferencias entre los países (12). Entre 1950 y 1990, en todos los países y regiones analizados se garantizaba el remplazo poblacional (por encima de dos hijos por mujer, en promedio). Entre 1990 y 1995, los países desarrollados y Cuba ya se encontraban por debajo de la cifra de remplazo; se estima que América Latina estará en una situación similar en la etapa 2030-2035. Cuba muestra las cifras más bajas de este indicador desde 1990 (cuadro 1), lo que evidencia la celeridad con que se ha reducido la tasa general de fecundidad en el país $(13,14)$.

La esperanza de vida al nacer. En contraste con el indicador anterior, este reveló una tendencia al ascenso entre 1950 y el 2015, con los mejores valores comparativos en Cuba a partir de 1990 (cuadro 1), aunque tuvo variaciones en los países (12). Se estima que este indicador - que refleja los años que en promedio se espera que vivan los integrantes de una generación- continúe en ascenso en todos los países y supere los 80 años para los que nazcan en el período 2045-2050 (1214). En el 2015, América Latina presentó avances significativos en la esperanza de vida, pues en todos los países, excepto en Bolivia y Haití, este indicador rebasó los 70 años $(12,15)$.

La esperanza de vida a la tercera edad. Este indicador refleja los años adicionales que en promedio se estima que vivan las personas que arribaron a los 60 años en un momento dado. Entre 1950 y 1955 era de 15,36 años en América Latina, 16,84 en los países desarrollados y 16,04 en Cuba (cuadro 1). Entre 1955 y el 2015, hubo un incremento, aunque variable, en los grupos de países analizados (12-15). Se espera que esta situación continúe en las décadas sucesivas hasta el 2050, 
aunque a ritmos más lentos. Esto confirma que el proceso de envejecimiento seguirá en ascenso.

Como resultado de estos cambios, la proporción de la población de 60 años o más ha aumentado en todos los países y regiones (cuadro 2): en Cuba se incrementó en 10 puntos porcentuales, en América Latina en 4,2 y en los países desarrollados en 10,3. En un horizonte temporal hasta el 2030, se vaticina un incremento de 12,7 puntos porcentuales en la proporción de personas mayores en Cuba y de 6,9 en los restantes países; esta proporción continuará en ascenso, al menos, hasta el 2050 (12-15). Ya en el 2010, Cuba, los países desarrollados y algunos latinoamericanos tenían más de $15 \%$ de la población con 60 años o más, lo que se considera un envejecimiento avanzado $(2,12)$. Para el 2030 se espera que los restantes países entren en esta categoría (1215). Los gobiernos de América Latina deben considerar la rapidez con que este proceso está ocurriendo, ya que es un desafío que la sociedad y la salud pública deberán afrontar en los próximos años.

En 1950, la proporción de personas de 75 años o más era pequeña (cuadro 2). Sin embargo, en el período estudiado se incrementó hasta $5 \%$ en los países desarrollados, $3,9 \%$ en Cuba y 1,7\% en América Latina. Se prevé que hacia el 2045, este grupo de edad seguirá creciendo (12-15).

\section{La transición epidemiológica}

Para los salubristas, es necesario añadir a este análisis otras aristas, como la transición epidemiológica $(4,8)$, la cual discurre de manera continua y explica el cambio en los patrones de mortalidad, morbilidad, fecundidad y en la organización de los servicios de salud, estrechamente relacionados con los eventos económicos (7). Este constructo se ha ido perfeccionando, en consonancia con nuevos enfoques teóricos y con la dinámica del contexto en que se inserta.

En la actualidad, se considera que las transiciones demográfica y epidemiológica son dos partes de un proceso continuo, en el que los patrones de salud y enfermedad de una sociedad se van transformando en respuesta a cambios más amplios de carácter demográfico, socioeconómico, tecnológico, político, cultural y biológico, por lo que ambas partes deben analizarse en estrecha relación. Este enfoque engloba la transición en las condiciones de salud que definen el perfil epidemiológico
CUADRO 1. Evolución histórica y proyecciones de la fecundidad, según indicadores de regiones y países seleccionados. Período 1950-2050

\begin{tabular}{|c|c|c|c|}
\hline \multirow{2}{*}{ Indicador y período } & \multicolumn{2}{|c|}{ Grupo de comparación } & \multirow{2}{*}{ Cuba } \\
\hline & Países desarrollados ${ }^{a}$ & América Latina & \\
\hline \multicolumn{4}{|l|}{ Tasa general de fecundidad } \\
\hline $1950-1955$ & 2,83 & 5,86 & 4,01 \\
\hline $1970-1975$ & 2,15 & 5,02 & 3,47 \\
\hline $1990-1995$ & 1,67 & 3,02 & 1,65 \\
\hline $2010-2015$ & 1,68 & 2,18 & 1,45 \\
\hline $2030-2035$ & 1,82 & 1,90 & 1,57 \\
\hline $2045-2050$ & 1,85 & 1,83 & 1,66 \\
\hline \multicolumn{4}{|l|}{ Esperanza de vida al nacer } \\
\hline $1950-1955$ & 64,67 & 51,37 & 59,40 \\
\hline $1970-1975$ & 71,08 & 61,03 & 70,98 \\
\hline $1990-1995$ & 74,09 & 68,93 & 74,79 \\
\hline $2010-2015$ & 77,73 & 74,70 & 78,45 \\
\hline 2030-2035 & 80,85 & 79,10 & 82,44 \\
\hline $2045-2050$ & 82,82 & 81,76 & 84,31 \\
\hline \multicolumn{4}{|c|}{ Esperanza de vida a la tercera edad ${ }^{b}$} \\
\hline $1950-1955$ & 16,84 & 15,36 & 16,04 \\
\hline $1970-1975$ & 18,15 & 17,52 & 19,05 \\
\hline 1990-1995 & 20,03 & 19,36 & 20,47 \\
\hline $2010-2015$ & 22,60 & 21,86 & 22,58 \\
\hline $2030-2035$ & 24,61 & 24,09 & 25,18 \\
\hline $2045-2050$ & 25,84 & 25,60 & 26,56 \\
\hline
\end{tabular}

a Para los efectos de este artículo, países desarrollados son los que han logrado un alto grado de industrialización, según los principales indicadores: producto interno bruto (PIB) nominal per cápita superior a US\$25000 en el 2005, PIB — según la paridad del poder adquisitivo per cápita— superior a US\$25 000 en el 2005 e índice de desarrollo humano superior a 0,900 en el 2004 (http://www.un.org/en/development/desa/publications/world-population-prospectsthe-2012-revision.html).

${ }^{\mathrm{b}}$ Años que, en promedio, se espera que vivan las personas que arriban a los 60 años en un período dado.

Fuente: Elaborado por los autores a partir de los datos de los anuarios estadísticos de salud, registros de mortalidad y fecundidad y de la referencia 12.

CUADRO 2. Evolución histórica y proyecciones de la proporción de personas mayores. Años, países y regiones seleccionados, período 1950-2045

\begin{tabular}{|c|c|c|c|}
\hline \multirow{2}{*}{ Indicador y año } & \multicolumn{2}{|c|}{ Grupos de comparación } & \multirow{2}{*}{ Cuba } \\
\hline & Países desarrollados ${ }^{a}$ & América Latina & \\
\hline \multicolumn{4}{|c|}{ Porcentaje de población de 60 años o más } \\
\hline 1950 & 11,5 & 5,6 & 7,0 \\
\hline 1970 & 14,6 & 6,3 & 9,1 \\
\hline 1990 & 17,7 & 7,3 & 12,0 \\
\hline 2010 & 21,8 & 9,8 & 17,0 \\
\hline 2030 & 28,7 & 16,7 & 29,7 \\
\hline 2045 & 31,4 & 23,0 & 36,3 \\
\hline \multicolumn{4}{|c|}{ Porcentaje de población de 75 años o más } \\
\hline 1950 & 2,4 & 1,0 & 1,3 \\
\hline 1970 & 3,4 & 1,2 & 1,6 \\
\hline 1990 & 5,4 & 1,7 & 3,7 \\
\hline 2010 & 7,4 & 2,7 & 5,2 \\
\hline 2030 & 10,9 & 4,9 & 9,5 \\
\hline 2045 & 13,9 & 8,2 & 15,2 \\
\hline
\end{tabular}

a Para los efectos de este artículo, países desarrollados son los que han logrado un alto grado de industrialización, según los principales indicadores: producto interno bruto (PIB) nominal per cápita superior a US\$25 000 en el 2005, PIB según la paridad del poder adquisitivo per cápita— superior a US\$25 000 en el 2005 e índice de desarrollo humano superior a 0,900 en el 2004 (http://www.un.org/en/development/desa/publications/world-population-prospects-the-2012revision.html).

Fuente: Elaborado por los autores a partir de las referencias 12 y 13. 
de una población (denominada transición estricta o transición epidemiológica estricta) y la respuesta social organizada a dichas condiciones, a través del sistema de atención a la salud (denominada transición de la atención sanitaria) (8).

En Cuba, la varicela ocupaba el primer lugar en la morbilidad por enfermedades infectocontagiosas en el período 19702015 , con un incremento relativo general de $16,1 \%$. Sin embargo, un análisis hacia el interior de la serie constata un comportamiento irregular de este indicador, con una tendencia al aumento en 1990, probablemente relacionado con el inicio de la crisis económica profunda que estaba sufriendo el país, y un descenso mantenido en los años 2010 y 2015 (cuadro 3). El sarampión se ubicó en el segundo lugar en 1970, pero no se han notificado casos nuevos desde 1993. La hepatitis viral ocupó el tercer lugar a inicios de la serie temporal analizada y, aunque tuvo un discreto incremento en 1990, experimentó una reducción marcada en el 2015, con una disminución relativa de $96,2 \%$ en el período. La parotiditis ocupó el cuarto lugar en 1970 y su incidencia se redujo ininterrumpidamente; desde el 2011 no se registran casos. Aunque la tasa se redujo en el período, la tuberculosis se mantiene entre las primeras causas de morbilidad, mientras que la incidencia de sífilis ha mostrado variaciones en los años de estudio. A pesar de la falta de datos para el período señalado, se constata que la tasa de tuberculosis en Cuba en el 2013 estuvo muy por debajo de la registrada en los países latinoamericanos, solo mejorada por Puerto Rico. Además, para esa fecha, el país presentaba una situación muy favorable en los indicadores de incidencia de la malaria, el dengue, la infección por el virus de la inmunodeficiencia humana (VIH) y el sida (14).

La vacunación en la infancia desempeñó un papel fundamental en la reducción de un grupo de enfermedades prevenibles a partir de 1959, entre las que se encuentran las mencionadas. En general, desde la década de 1990 se observa una reducción - tanto absoluta como relativa- de las enfermedades infectocontagiosas $y$ un predominio de las enfermedades no transmisibles (3, 12-14).

Hoy se conoce el impacto positivo de la prevención temprana de las enfermedades infectocontagiosas sobre la

CUADRO 3. Morbilidad por las principales enfermedades infectocontagiosas. ${ }^{a}$ Cuba, años seleccionados

\begin{tabular}{llrr}
\hline \multicolumn{1}{c}{ Año y No. de orden } & Causa & Casos notificados & Tasa $^{\text {b }}$ \\
\hline 1970 & Varicela & 12714 & 148,3 \\
1 & Sarampión & 8911 & 104,2 \\
2 & Hepatitis & 8692 & 101,4 \\
3 & Parotiditis & 2820 & 32,9 \\
4 & Tuberculosis & 2606 & 30,5 \\
5 & & & \\
1990 & Varicela & 37583 & 354,4 \\
1 & Hepatitis & 13214 & 124,6 \\
2 & Tuberculosis & 546 & 5,1 \\
3 & Leptospirosis & 517 & 4,9 \\
4 & Enfermedad meningocócica & 449 & 4,2 \\
5 & & & \\
2010 & Varicela & 32377 & 1288,1 \\
1 & Sífilis & 1445 & 11,2 \\
2 & Hepatitis & 1261 & 7,0 \\
3 & Tuberculosis & 782 & \\
4 & & & 172,2 \\
2015 & Varicela & 19330 & 40,8 \\
1 & Sífilis & 4582 & 3,9 \\
2 & Hepatitis & 433 & 5,8 \\
3 & Tuberculosis & 651 & \\
4 & Fuente: Elaborado por los autores a partir de los datos de los anuarios estadísticos de salud publicados por el Ministerio \\
de Salud Pública. & & &
\end{tabular}

esperanza de vida, y su contribución al incremento poblacional de los grupos de mayor edad (16). Por ello, deberá considerarse la administración de vacunas profilácticas dirigidas a las personas mayores, afectadas por la llamada inmunosenescencia.

A partir de 1990, con el aumento del número de personas mayores, se incrementó la prevalencia de las enfermedades crónicas (figura 1). Con respecto a 1990, en el 2015 se observó un aumento relativo en la prevalencia de la hipertensión arterial de $239,0 \%$, la diabetes mellitus de 192,3\% y el asma bronquial de $114,3 \%(3,12-14)$. El análisis integrado del cuadro 3 y la figura 1 muestra que en la medida en que la morbilidad por enfermedades infectocontagiosas se reducía de manera importante, la prevalencia de las crónicas aumentaba. Esto se corresponde con el envejecimiento acelerado de la población cubana y las políticas sociales y sanitarias establecidas en el país desde mediados del siglo XX, concretadas en los planes y los programas de salud (2).

Durante el período 1970-2015, se mantuvieron las primeras causas de muerte en Cuba (figura 2). Sin embargo, se observó un ascenso en la mortalidad por tumores malignos y una ligera reducción en la originada por enfermedades del corazón, mientras que la mortalidad por enfermedades cerebrovasculares se mantuvo estable. Esta estructura de la mortalidad es similar a la de los países desarrollados, en los que el envejecimiento poblacional tiene un peso importante (13).

\section{AFRONTAMIENTO DEL ENVEJECIMIENTO DE LA POBLACIÓN EN CUBA: UNA VISIÓN DESDE LA SALUD PÚBLICA}

El envejecimiento poblacional impone retos económicos y sociales considerables, ya que el Estado debe garantizar una atención óptima a las personas mayores. Esta tarea debe recaer sobre todos los sectores de la sociedad, a fin de, en conjunto, contribuir a la prolongación de la vida y a una mayor calidad de esta.

El nuevo modelo económico cubano prioriza la atención a las personas mayores y dedica varios de sus lineamientos a ellas (17). Como el envejecimiento poblacional exige una respuesta integral de salud pública, se hace necesario adaptar los 
FIGURA 1. Tasa de prevalencia de las principales enfermedades crónicas no transmisibles. Cuba, años seleccionados

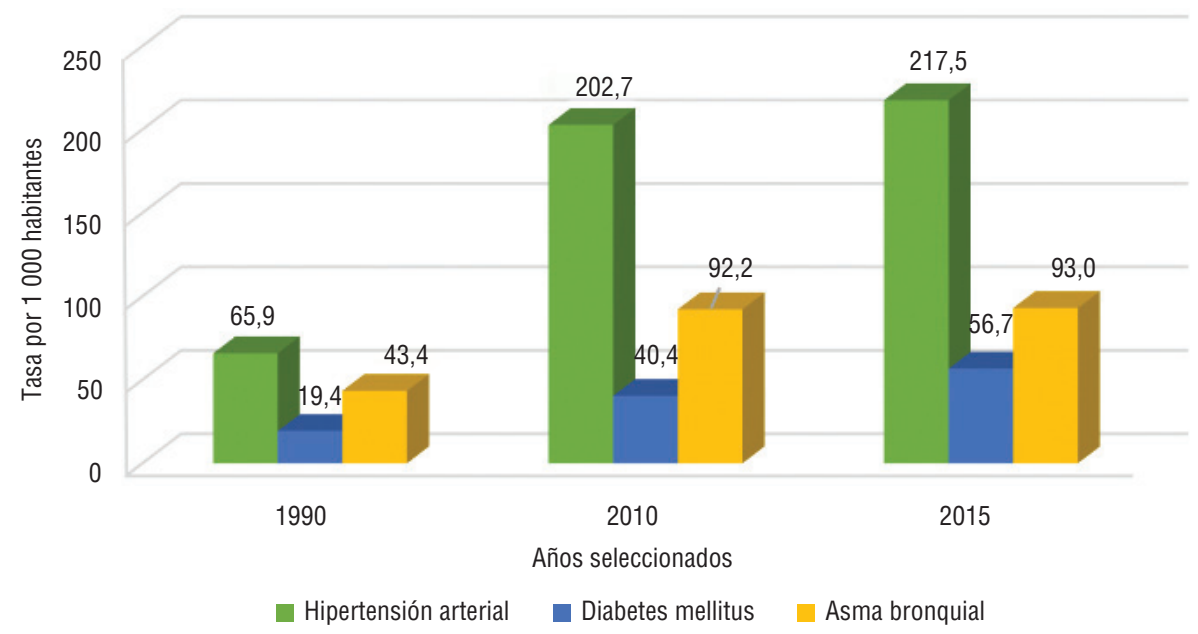

Fuente: Elaborado por los autores a partir de los datos de los anuarios estadísticos de salud publicados por el Ministerio de Salud Pública.

FIGURA 2. Tasa de mortalidad en las personas mayores según las causas principales. Cuba, años seleccionados

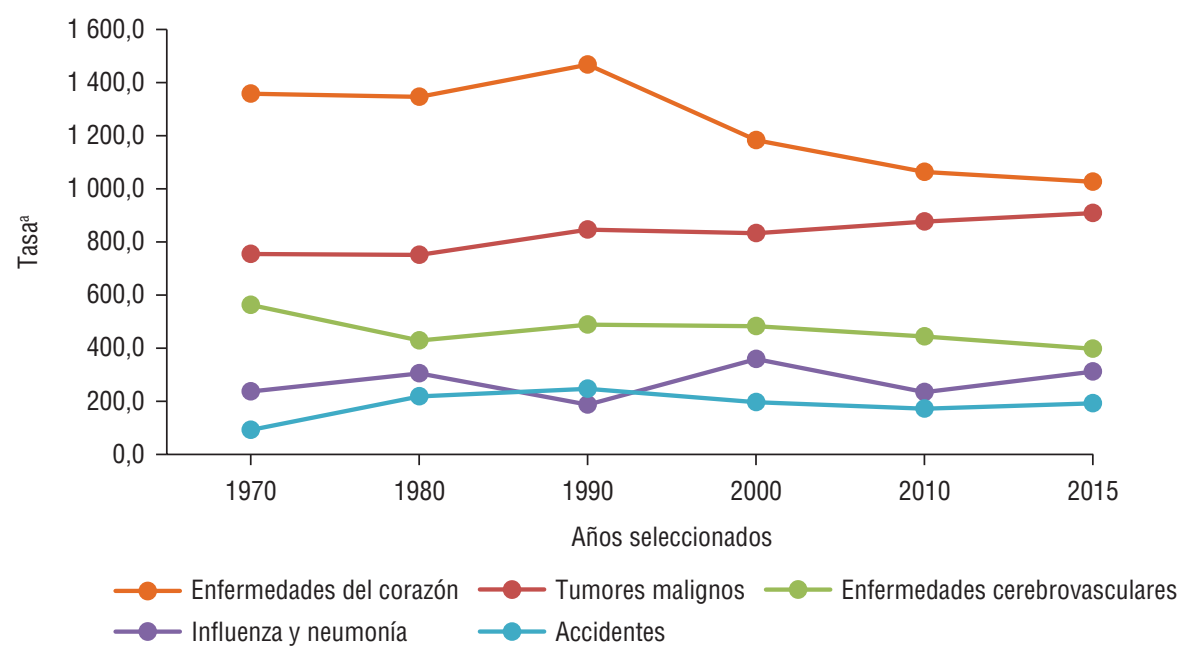

Tasa por 10000 habitantes de 60 años o más.

Fuente: Elaborado por los autores a partir de los datos de los anuarios estadísticos de salud publicados por el Ministerio de Salud Pública.

sistemas de salud a los requerimientos, las expectativas y las preferencias de las personas mayores. Además, se requiere fomentar iniciativas coordinadas entre todos los sectores y promover entornos amigables (18). La política para el sector sanitario contempla la estrategia de atención primaria de salud como base para lograr un envejecimiento saludable, el establecimiento de cuidados a largo plazo y al final de la vida a nivel comunitario, el incremento del número de la servicios y especialistas de geriatría en el país, y la capacitación de los profesionales de la salud en materia de envejecimiento.
Pasar la vejez en el lugar donde ha transcurrido la vida facilita a las personas mayores los lazos con su comunidad y las redes sociales. Asimismo, procura empoderarlas para que contribuyan a la sociedad y sigan siendo miembros activos de sus comunidades (19). Una de las acciones encaminadas a lograr el envejecimiento saludable es la incorporación de estas personas a los llamados círculos de abuelos, una iniciativa comunitaria que contribuye a la práctica de la actividad física, la diseminación de información sobre estilos de vida saludable, la realización de actividades sociales y cul- turales, la participación social y el bienestar de sus integrantes.

Los profesionales que trabajan en el nivel primario cuentan con una guía práctica para la evaluación y la atención de los problemas de salud más frecuentes en el ámbito comunitario: caídas, demencia, depresión, incontinencia urinaria, dolor persistente, administración de medicamentos, entre otros. En el 2016, se logró un promedio de 1,2 exámenes periódicos al año por persona mayor, lo que supera el estándar aceptado de un examen al año.

La vejez genera nuevas necesidades de cuidado que deben ser consideradas en la familia y en la organización social. Según los enfoques actuales, los cuidados a largo plazo deben ofrecerse en diversos ámbitos: el domicilio, los centros comunitarios, los hogares para personas mayores, los hospitales y otras instituciones de salud (20). En este sentido, la preparación de los cuidadores es otro de los desafíos que impone el envejecimiento de la población.

Cuba cuenta también con las llamadas casas de abuelos, centros comunitarios que brindan atención integral diurna a las personas mayores solas o sin familiares que puedan atenderlos durante el día, y que solo presenten dificultades para realizar algunas actividades instrumentales de la vida diaria. En el 2016, funcionaban 276 casas de abuelos, con capacidad para atender 8217 personas (6). También se han creado casas de abuelos especializadas para la atención a personas mayores con demencia leve o moderada. Otros centros de atención son los hogares de ancianos, donde se ofrece el cuidado institucional de corta, mediana y larga estadía a las personas con dependencia moderada y grave que no se benefician con otras alternativas en la comunidad. En el país funcionan 148 hogares de ancianos, que atienden en régimen de internamiento a 4,5 por cada 10000 personas mayores (6).

Sin embargo, la responsabilidad de los cuidados a largo plazo recae principalmente en la familia a través de los cuidadores primarios; la mayoría de ellos son mujeres, casi siempre hijas o nueras sin experiencia en la atención a personas mayores dependientes. Esto puede reflejarse negativamente en la calidad del cuidado que se brinda y en la salud del cuidador, a lo que se adiciona la pérdida de su vínculo laboral. Esto afecta a la economía individual y fami- 


\title{
CUADRO 4. Lecciones aprendidas en el proceso de afrontamiento del envejecimiento demográfico en Cuba
}

\begin{tabular}{|c|c|}
\hline Dificultad & Solución \\
\hline $\begin{array}{l}\text { Disminución del número de personas en edad productiva, lo } \\
\text { que genera incremento del índice de dependencia, } \\
\text { fundamentalmente a expensas de las personas mayores }\end{array}$ & $\begin{array}{l}\text { - Prolongación a cinco años más de la edad de jubilación para ambos sexos } \\
\text { - Aumento del monto de las pensiones de acuerdo con los años de trabajo } \\
\text { - Oferta de reinserción laboral a jubilados }\end{array}$ \\
\hline Reducción de la natalidad por debajo del nivel de remplazo & $\begin{array}{l}\text { - Cambios en las legislaciones sobre la maternidad para incentivar los nacimientos, como prolongar el } \\
\text { tiempo de licencia de trabajo, ofrecer pagos, reconocer la solicitud de licencia de trabajo del padre y la } \\
\text { diferenciación de beneficios en dependencia del número de hijos }\end{array}$ \\
\hline $\begin{array}{l}\text { Incremento de la morbilidad por enfermedades no } \\
\text { transmisibles }\end{array}$ & $\begin{array}{l}\text { - Promoción de estilos de vida saludable a través de los círculos de abuelos y otras medidas incluidas dentro } \\
\text { de la estrategia de atención primaria de salud } \\
\text { - Acercamiento de los servicios especializados a este grupo de población, como las salas de rehabilitación } \\
\text { integral } \\
\text { - Aumento del número de servicios y especialistas en geriatría en todos los niveles de atención } \\
\text { - Programas verticales de atención a las enfermedades no transmisibles de mayor prevalencia } \\
\text { - "Geriatrización" de los serviciosa }\end{array}$ \\
\hline $\begin{array}{l}\text { Aumento del número de personas mayores solas y } \\
\text { discapacitadas }\end{array}$ & $\begin{array}{l}\text { - Perfeccionamiento de la atención institucional a este tipo de anciano (hogares de ancianos y casas de } \\
\text { abuelos) } \\
\text { - Cambios en la asistencia social: asignación de cuidadores remunerados, capacitación a los cuidadores } \\
\text { informales (escuelas de cuidadores), ayudas técnicas por el estado, creación de servicios subsidiados } \\
\text { como la alimentación, y la implementación de la Estrategia Cubana contra la Enfermedad de Alzheimer y } \\
\text { los Síndromes Demenciales }\end{array}$ \\
\hline
\end{tabular}

\begin{abstract}
a Aporte de competencias sobre temas relacionados con la atención a los problemas principales que afectan a la salud de las personas mayores a otros profesionales de la salud no especializados en geriatría, profesionales de otros sectores, estudiantes de ciencias médicas, así como a las familias y a la comunidad, con vistas a mejorar la atención integral a este segmento de la población.
\end{abstract}

liar, además del costo social, que se estima en una pérdida del 2,2\% del PIB (21). De ahí que al análisis económico tradicional se debe incorporar la economía del cuidado y los desafíos impuestos por la conciliación de la vida laboral con la familiar (22).

La atención y el apoyo a los cuidadores primarios es un objetivo prioritario de la estrategia gubernamental para afrontar el envejecimiento. Para brindar cuidado se necesitan conocimientos, habilidades, valores y experiencia, y es fundamental proporcionar a estas personas la formación que les permita hacer bien su trabajo. En Cuba existe un programa psicoeducativo para facilitadores llamado "Cuidar a quien nos cuida", que forma parte de la iniciativa Escuela de Cuidadores en el Nivel Primario, basado en los recursos humanos y la infraestructura disponibles en la comunidad. Este programa combina las estrategias de cuidado con la vida laboral del cuidador, estimula el desarrollo de competencias para la vida, fomenta el autocuidado y persigue disminuir la carga del cuidador, garantizar los derechos de las personas mayores y sus cuidadores, y fortalecer los servicios y las redes de apoyo comunitarios. Existen 396 escuelas de cuidadores y se elaboró el Manual para cuidadores de adultos mayores dependientes, como material educativo complementario, que ofrece información práctica para la atención de estas personas.

En la actualidad, la atención sanitaria a las personas mayores supone el
$28 \%$ de la carga de trabajo en la atención primaria, $32 \%$ de la carga en cirugía, $43 \%$ en las especialidades médicas y $48 \%$ de las hospitalizaciones (21). Los profesionales de la salud deben contar con las competencias adecuadas para satisfacer las demandas y las necesidades de salud de las personas mayores. Al respecto, se han realizado acciones dirigidas a garantizar que el personal de la salud esté debidamente capacitado: cursos básicos, entrenamientos y diplomados para profesionales que laboran en el Sistema Nacional de Salud; además, se introdujeron temas relacionados con el envejecimiento en los planes de estudios de las carreras de medicina y enfermería.

Es indispensable garantizar que el número de geriatras satisfaga las necesidades de la población adulta mayor. En la actualidad hay 441 especialistas graduados o en formación, lo que representa 2 especialistas por cada 10000 personas mayores. Si se toman en cuenta otros médicos dedicados a esta actividad, el indicador asciende aproximadamente a 5 médicos por cada 10000 personas mayores, que en el 2016 brindaron 6,6 consultas por persona mayor (6). En los hospitales existen 50 servicios de geriatría, con una cama por cada 2000 personas mayores; en el 2016 estos servicios registraron 1,4 ingresos hospitalarios por cada 1000 personas mayores (6).

Se han elaborado los protocolos de actuación para los principales problemas de salud de este grupo: caídas, inconti- nencia urinaria, deterioro cognitivo y demencias, inmovilidad, estado confusional agudo, hipertensión arterial, insuficiencia cardíaca, anemia, enfermedad pulmonar obstructiva crónica y cáncer. En la actualidad se cuenta con 18 servicios especializados para el diagnóstico, el tratamiento y la rehabilitación de los síntomas psíquicos y motores de la demencia.

Dada la magnitud y la gravedad de las demencias, se aprobó la Estrategia Cubana para la Enfermedad de Alzheimer y los Síndromes Demenciales (23).

La atención del envejecimiento en Cuba ha requerido de soluciones a los principales problemas que este proceso ha originado para el país (cuadro 4).

\section{CONCLUSIONES Y RECOMEN- DACIONES}

El envejecimiento demográfico acelerado que ocurre en Cuba, cuyo origen está en la reducción sostenida de los niveles de fecundidad y mortalidad, y el saldo migratorio negativo, representa un logro y una oportunidad de desarrollo, pero constituye un desafío socioeconómico y de salud pública.

El afrontamiento al envejecimiento acelerado de la población cubana se realiza mediante la implementación de políticas y estrategias integrales e intersectoriales, contextualizadas al país, para mitigar los efectos negativos del proceso. En estas, el sector de la salud desempeña un papel protagónico, dada la repercusión que el incremento de la 
demanda de los servicios de salud de alto coste pudiera representar sobre la organización y la calidad de los servicios que el sector brinda.

Se recomienda continuar con el estudio sistemático y la monitorización del envejecimiento en Cuba, que aporten las evidencias necesarias para la toma de decisión oportuna y eficiente por parte de los directivos del sector para mantener la calidad de los servicios a esta población.

\section{Conflicto de intereses. Ninguno.}

\section{REFERENCIAS}

1. Bayarre Vea H. Múltiples perspectivas para el análisis del envejecimiento demográfico. Una necesidad en el ámbito sanitario contemporáneo. Rev Cubana Salud Pública. 2017;43(2):314-6. Disponible en: http: / / www.revsaludpublica.sld.cu / index.php/spu/article/view/892/928 Acceso el 10 de abril de 2017.

2. Aja Díaz A, Catasús Cervera SI, Benítez Pérez ME, San Marful Orbis E, Oliveros Blet A, Rodríguez Gómez G, et al. Características sociodemográficas de la población cubana. La Habana: Centro de Estudios Demográficos; 2012.

3. Alfonso Fraga JC, Mena Corre M, Franco Suárez MC, Plana Álvarez A, Pifferrer Campins G, de la Caridad Hernández E, et al. Envejecimiento poblacional en Cuba. La Habana: Ciencias Sociales; 2016.

4. Bayarre Vea HD, Pérez Piñero JS, Menéndez Jiménez J. Las transiciones demográfica y epidemiológica y la calidad de vida objetiva en la tercera edad. Gero Info. 2006;1(3). Disponible en: http:/ /www.sld. $\mathrm{cu} /$ galerias/pdf//sitios/gericuba/las_ transiciones_demografica y_epidemiologica_y_la_calidad_de_vida_ objetiva_en_la_tercera_edad.pdf Acceso el 7 de marzo de 2017.

5. Cabrera Marrero F. Transición demográfica y transición epidemiológica: ¿Hacia dónde transita el Caribe? Novedades Poblacion (La Habana). 2011;39(2):71-9.

6. Cuba, Ministerio de Salud Pública. Anuario Estadístico de Salud 2016. La Habana: Dirección Nacional de Estadística; 2017. Disponible en: http://files.sld.cu/dne/ files/2017/05/Anuario_Estad\%C3\%ADstico_de_Salud_e_2016_edici\%C3\%B3n_2017. pdf Acceso el 14 de abril de 2017.

7. Louro Bernal I, Bayarre Vea H, Álvarez Lauzarique ME. Implicaciones familiares y sanitarias del envejecimiento poblacional en la cobertura universal. Rev Cubana Salud Publica. 2015;41(Supl 1):80-96. Disponible en: http://www.revsaludpublica. sld.cu/index.php/spu/article / view/638/527 Acceso el 10 de abril de 2017.

8. Frenk J, Frejka T, Bobadilla JL, Stern C, Lozano R, Sepúlveda JM. The epidemiological transition in Latin America.
Bol Of Sanit Panam. 1991;111(6):485-96. Disponible en: http://www.incap.paho. org/portaleducativo/index.php/es/recursos / reservorio-san / doc_view / 492-3-la-transicion-epidemiologica Acceso el 22 de febrero de 2017.

9. Rodríguez Gómez G, Albizu-Campos Espiñeira JC. La dinámica demográfica cubana en el contexto actual. En: Espina MP, Echevarría D. Cuba: los correlatos socioculturales del cambio. La Habana: Ciencias Sociales; 2015.

10. Albizu-Campos Espiñeira JC, de Almeida Rezende DF. Dinámica demográfica cubana. Antecedentes para un análisis. Novedades Poblacion (La Habana). 2014;42(6):4-31.

11. Rodríguez Gómez G. De lo individual a lo social: cambios en la fecundidad cubana. La Habana: Centro de Estudios Demográficos; 2013.

12. United Nations. World Population Prospects. The 2012 revision. Highlights and advance tables. New York: UN Department of Economics and Social Affairs, Population Division; 2013. Disponible en: https: / / esa.un.org/unpd/wpp/publications/Files/WPP2012_HIGHLIGHTS.pdf Acceso el 10 de septiembre de 2017.

13. Zacca Peña E, Gran Álvarez MA, Torres Vidal RM, Alonso Alomá I, Martínez Morales MA, Felipe Ramos AM, et al. Temas estadísticos de salud. La Habana: Ministerio de Salud Pública; 2011.

14. Cuba, Ministerio de Salud Pública. Anuario Estadístico de Salud 2015. La Habana: Dirección Nacional de Estadística; 2016.

15. Organización Panamericana de la Salud. Situación de salud en las Américas. Indicadores básicos 2015. Washington, DC: OPS; 2016. Disponible en: http://www.gestars a l u d . com / w p - con t e n t / uploads/2016/03/Situaci\%C3\%B3n-deSalud-en-las-Am\%C3\%A9ricas-Indicadores-B\%C3\%A1sicos-de-Salud-2015.pdf Acceso el 17 de marzo de 2017.

16. Rojas Ochoa F. Vacunas. Cuba 1959-2008. La Habana: Editorial Ciencias Médicas; 2011.

17. Partido Comunista de Cuba. Actualización de los Lineamientos de la Política Económica y Social del Partido y la Revo-
Declaración. Las opiniones expresadas en este manuscrito son responsabilidad de los autores y no reflejan necesariamente los criterios ni la política de la Revista Panamericana de Salud Pública / Pan American Journal of Public Health o de la Organización Panamericana de la Salud. lución para el período 2016-2021. La Habana: Editora Política; 2016. Disponible en: http://www.granma.cu/file/pdf/gaceta/01Folleto.Lineamientos-4.pdf Acceso el 22 de septiembre de 2017.

18. Torrado Ramos AM, Sánchez Pérez L, Somonte López R, Cabrera Marsden AM, Hernández Pino PC, Lorenzo Pérez O. Envejecimiento poblacional: una mirada desde los programas y políticas públicas de América Latina, Europa y Asia. Novedades Poblacion (La Habana). 2014;42(7):2634.

19. Organización Mundial de la Salud. Informe mundial sobre el envejecimiento y la salud. Ginebra: OMS; 2015.

20. Abizanda Soler P, Sáez Méndez L, Cano Gutiérrez CA. Formación y docencia en geriatría. Capítulo 11. En: Abizanda Soler P. ed. Tratado de Medicina Geriátrica. Fundamentos de la atención sanitaria a los mayores. Madrid: Elsevier; 2015. Pp. 90-3.

21. Hernández Montero A, Castiñeiras García $\mathrm{R}$, Menéndez Jiménez J. Proceso de envejecimiento y su impacto en el desempeño económico: una aproximación. Cuba Invest Econ. 2016;22(2):9-32.

22. Lara Junco T. Economía del cuidado en tiempos de transformaciones económicas. En: Pérez Villanueva OE, Torres Pérez R. Miradas a la economía cubana. Entre la eficiencia económica y la equidad social. La Habana: Editorial Caminos; 2013. Pp. 147-60.

23. Bosh Bayard RL, Llibre Rodríguez JJ, Fernández Seco A, Borrego Calzadilla C, Carrasco García M, Zayas Llerena T, et al. Cuba's strategy for Alzheimer disease and dementia syndromes. MEDICC Rev. 2016;18(4):9-12. Disponible en: http:// www.redalyc.org/articulo.oa?id= 437548048002 Acceso el 10 de septiembre de 2017.

Manuscrito recibido el 27 de abril de 2017. Aceptado para publicación, tras revisión, el 30 de noviembre de 2017. 
ABSTRACT The demographic aging in Cuba has caused considerable changes in mortality and morbidity; its growing trend and high speed affect the attention of the elderly, posing a challenge to public health. This article focuses on years 1950-2015, and aims to

\section{Approaches, evolution and coping with the demographic aging in Cuba}

Keywords present the situation of this population phenomenon from a positive view, according to three aspects: aging as an achievement, challenge and development opportunity; demographic and epidemiological transitions; and policies and strategies to address aging. The general fertility rate has decreased from 4.01 to 1.45, and the life expectancy at birth and the life expectancy at third age have increased from 59.40 to 78.45 years and from 16.04 to 22.58 , respectively. The proportion of people aged 60 and over in Cuba amounts to $19.8 \%$. Causes of morbidity and mortality have changed from infectious diseases to non-communicable diseases. In Cuba, aging is the result of a sustained reduction in fertility and mortality, and a negative migratory balance. The challenge of the dependency of the elderly person and the need for care is discussed. Although this demographic indicator represents an achievement and a development opportunity, population aging poses a challenge for development and public health, for which comprehensive and cross-sectional policies and strategies aimed at mitigating its negative effects have been established.

Demographic aging; public health; demographic transition; health transition; public policies; Cuba.
RESUMO

\section{Abordagens, evolução e enfrentamento do envelhecimento demográfico em Cuba}

Palavras-chave
O envelhecimento demográfico em Cuba causou mudanças consideráveis na mortalidade e morbidade; sua crescente tendência e alta velocidade comprometem a atenção dos idosos, portanto, é um desafio para a saúde pública. Este artigo, que abrange o período 1950-2015, visa expor a situação deste fenômeno da população de uma visão positiva, de acordo com três aspectos: o envelhecimento como realização, desafio e oportunidade de desenvolvimento; transições demográficas e epidemiológicas; e as políticas e estratégias para enfrentá-lo. A taxa geral de fertilidade diminuiu de 4,01 para 1,45 , e a expectativa de vida no nascimento e a expectativa de vida na terceira idade aumentaram de 59,40 para 78,45 anos e de 16,04 a 22,58 , respectivamente. A proporção de pessoas com 60 e mais anos em Cuba é de 19,8\%. O quadro de morbidade e mortalidade mudou de doenças infecciosas para doenças não transmissíveis. $\mathrm{O}$ envelhecimento em Cuba foi produzido pela redução sustentada da fertilidade e da mortalidade, e um saldo migratório negativo. $\mathrm{O}$ desafio da dependência da pessoa idosa e a necessidade de cuidados que eles requerem são discutidos. Além da conquista e da oportunidade de desenvolvimento representada por esse indicador demográfico, o envelhecimento da população constitui um desafio para o desenvolvimento e a saúde pública, para o qual foram estabelecidas políticas e estratégias abrangentes e intersetoriais destinadas a mitigar seus efeitos negativos.

Envelhecimento da população; saúde pública; transição demográfica; transição epidemiológica; políticas públicas; Cuba. 